\title{
Exérese de mucocele em lábio inferior: relato de caso
}

\author{
Exeresis of lower lip mucocele: case report \\ Exéresis de mucocele en labio inferior: relato de caso
}

Recebido: 16/08/2021 | Revisado: 21/08/2021 | Aceito: 31/08/2021 | Publicado: 03/09/2021

Cristiane Sales da Costa

ORCID: https://orcid.org/0000-0001-9243-4807

Faculdades Integradas Aparício Carvalho, Brasil

E-mail: cris.sales7@gmail.com

Marcos Vinicius Montagna

ORCID: https://orcid.org/0000-0002-4961-851X

Faculdades Integradas Aparício Carvalho, Brasil

E-mail: marcos010248@gmail.com

Ilso Márcio Gedro Rocha

ORCID: https://orcid.org/0000-0002-9913-9445

Faculdades Integradas Aparício Carvalho, Brasil E-mail: prof.rocha.ilso@fimca.com.br

Flávio Martins da Silva

ORCID: https://orcid.org/0000-0003-2231-7634 Faculdades Integradas Aparício Carvalho, Brasil

E-mail: prof.flavio.martins@ fimca.com.br

Leopoldo Luiz Rocha Fujii

ORCID: https://orcid.org/0000-0003-0620-1236 Faculdades Integradas Aparício Carvalho, Brasil

E-mail: prof.leopoldo.fujii@fimca.com.br

Daniele Paraguassú Fagundes de Souza

ORCID: https://orcid.org/0000-0002-2786-498X

Faculdades Integradas Aparício Carvalho, Brasil

E-mail: daniele.souza@ fimca.com.br

Arlindo Tadeu Teixeira Aburad

ORCID: https://orcid.org/0000-0002-7601-0286 Laboratório de Patologia Bucal e Maxilofacial, Brasil

E-mail: bruno.c.mnds@gmail.com

Bruno Coelho Mendes

ORCID: https://orcid.org/0000-0001-7896-8909 Faculdades Integradas Aparício Carvalho, Brasil E-mail: brunocoelho.mnds@gmail.com

\begin{abstract}
Resumo
A mucocele oral é considerada a lesão benigna mais comum das glândulas salivares menores, correspondendo a um fenômeno de retenção ou extravasamento de muco. A mucocele oral se origina através de traumatismos mecânicos, e acomete na maioria das vezes o lábio inferior, por ser uma região mais propensa à traumas. Este artigo objetiva apresentar um caso clínico de uma paciente de 21 anos com queixa de aumento volumétrico em lábio inferior, com um ano de evolução e que não apresentou episódios de ruptura ou remissão. Sob a hipótese diagnóstica de mucocele, foi realizada a excisão da lesão em lábio inferior, onde a incisão mucosa foi precisamente delimitada com auxílio de um punch de biópsia e a lesão, juntamente com as glândulas salivares menores foram removidas por meio de dissecção com tesoura íris. O diagnóstico definitivo obtido após a análise histopatológica foi de mucocele, confirmando a hipótese apresentada inicialmente. O caso clínico apresentado obteve um resultado satisfatório com a técnica empregada, permitindo boa evolução, com ausência de infecção e de recidiva.
\end{abstract}

Palavras-chave: Mucocele; Lábio; Patologia bucal; Biópsia.

\section{Abstract}

Oral mucocele is considered the most common benign lesion of the minor salivary glands, corresponding to a phenomenon of retention or extravasation of mucus. Oral mucoceles originate through mechanical trauma, and most often affect the lower lip, as it is a region more prone to trauma. This article aims to present a clinical case of a 21 year-old patient complaining of volumetric increase in the lower lip, with one year of evolution and who did not present episodes of rupture or remission. Under the diagnostic hypothesis of mucocele, the lesion was excised in the lower lip, where the mucosal incision was precisely delimited with the aid of a biopsy punch and the lesion, with the minor salivary glands, were removed by dissection with iris scissors. The definitive diagnosis obtained after 
histopathological analysis was mucocele, confirming the hypothesis initially presented. The clinical case presented obtained a satisfactory result with the technique used, allowing good evolution, with no infection and no recurrence.

Keywords: Mucocele; Lip; Oral pathology; Biopsy.

\section{Resumen}

El mucocele oral se considera la lesión benigna más común de las glándulas salivales menores, correspondiente a un fenómeno de retención o extravasación de moco. Los mucoceles orales se originan a través de traumatismos mecánicos y, con mayor frecuencia, afectan el labio inferior, ya que es una región más propensa a traumatismos. Este artículo tiene como objetivo presentar un caso clínico de un paciente de 21 años que consulta por aumento volumétrico en el labio inferior, con un año de evolución y que no presentó episodios de rotura o remisión. Bajo la hipótesis diagnóstica de mucocele, la lesión se extirpó en el labio inferior, donde se delimitó con precisión la incisión de la mucosa con la ayuda de un punch de biopsia y se extirpó la lesión, junto con las glándulas salivales menores, mediante disección con tijeras de iris. El diagnóstico definitivo obtenido tras el análisis histopatológico fue de mucocele, confirmando la hipótesis inicialmente presentada. El caso clínico presentado obtuvo un resultado satisfactorio con la técnica empleada, permitiendo una buena evolución, sin infección y sin recidiva.

Palabras clave: Mucocele; Labio; Patología bucal; Biopsia.

\section{Introdução}

A mucocele oral, lesão benigna mais comum das glândulas salivares menores, corresponde a um fenômeno de extravasamento ou de retenção de muco, cuja etiologia está relacionada a um trauma local onde há o rompimento do ducto de uma glândula salivar menor. A lesão tipo extravasamento ocorre após o rompimento do ducto devido ao acúmulo excessivo de secreção, que, uma vez no epitélio, é encapsulada por tecido de granulação. Por sua vez, a lesão tipo retenção é caracterizada pelo revestimento por epitélio do ducto glandular, sem extravasamento. Esta ocorre com menos frequência e geralmente está localizada na bochecha ou palato de pacientes idosos (Jimbu et al, 2003; Choi, Byun, Choi, \& Jung, 2019).

Clinicamente pode ser observada como uma tumefação ou bolha, assintomática, flácida a palpação, de coloração semelhante à da mucosa adjacente ou azulada, dependendo da sua profundidade nos tecidos, com a superfície lisa e seu tamanho variado (Nico; Park; Lourenço, 2008; Khandewal \& Patil, 2012; Jimbu et al, 2019). Embora sejam assintomáticas, podem causar dificuldades na fala, mastigação e deglutição (Martinez et al, 2010). O lábio inferior é o local mais frequentemente afetado, por ser a região mais suscetível a traumas, em crianças e adultos jovens, tendo como principal fator associado a mordida acidental ou hábitos parafuncionais (Santos et al, 2008; Nallasivam \& Sudha, 2015; Bezerra, et al, 2016).

O diagnóstico clínico é baseado na história apresentada pelo paciente e na apresentação clínica da lesão. Para as mucoceles orais, pode-se considerar, no diagnóstico diferencial, o lipoma, fibroma e o papiloma (Valério, de Queiroz, Romualdo, Brentegani \& de Paula-Silva, 2013; Bansal, Verma, Goyal, \& Rai, 2017). A confirmação e obtenção do diagnóstico definitivo se dá através do exame histopatológico (Valério et al, 2013; Choi et al, 2019).

Segundo Oliveira, Henrique e Cruz, (2018) o tratamento pode incluir excisão cirúrgica da lesão pelo método convencional ou por meio do uso de laser de diodo, criocirurgia, escleroterapia, micromarsupialização, injeção intralesional de corticosteroide. Além disso, a lesão pode sofrer regressão espontânea. O meio mais comumente utilizado é a remoção cirúrgica convencional com auxílio de uma lâmina de bisturi, sendo necessária a exérese completa da lesão e glândulas salivares menores associadas para reduzir o risco de recidiva. O presente artigo relata a exérese de uma mucocele em lábio inferior com o auxílio de um punch para biópsia.

\section{Metodologia}

Um estudo intervencional descritivo e qualitativo foi realizado como relato de caso do tratamento e acompanhamento do paciente. A história da doença e exame físico do paciente foram coletados após autorização e assinatura do termo de consentimento livre e esclarecido, como (Rodrigues et al, 2021; Mendes, Bonardi, Silva, Crivelini \& Bassi, 2020; Silva et al, 2021). Este relato de caso, realizado com base em uma revisão simples da literatura, é apresentado com base nos descritores 
(Mucocele; Lábio; Patologia bucal; Biópsia). Para a realização do tratamento apresentado não foi necessário a aprovação pelo Comitê de ética, apenas a obtenção do Termo de Consentimento Livre e Esclarecido assinado pelo paciente.

\section{Relato de caso}

Paciente de 21 anos, feoderma, gênero feminino, saudável, procurou a clínica odontológica do Centro Universitário FIMCA, Porto-Velho, queixando-se de aumento volumétrico em mucosa do lábio inferior, próximo à comissura labial, com 12 meses de evolução e sem períodos de remissão ou ruptura.

O exame físico evidenciou tumefação arredondada na mucosa do lábio inferior no lado direito, assintomática, com dimensões aproximadas de $5 \mathrm{~mm}$, de coloração normal da mucosa, superfície lisa, com base séssil, sendo flácida à palpação (Figura 1).

Figura 1 - Lesão de aproximadamente 5mm em lábio inferior, com coloração normal da mucosa.

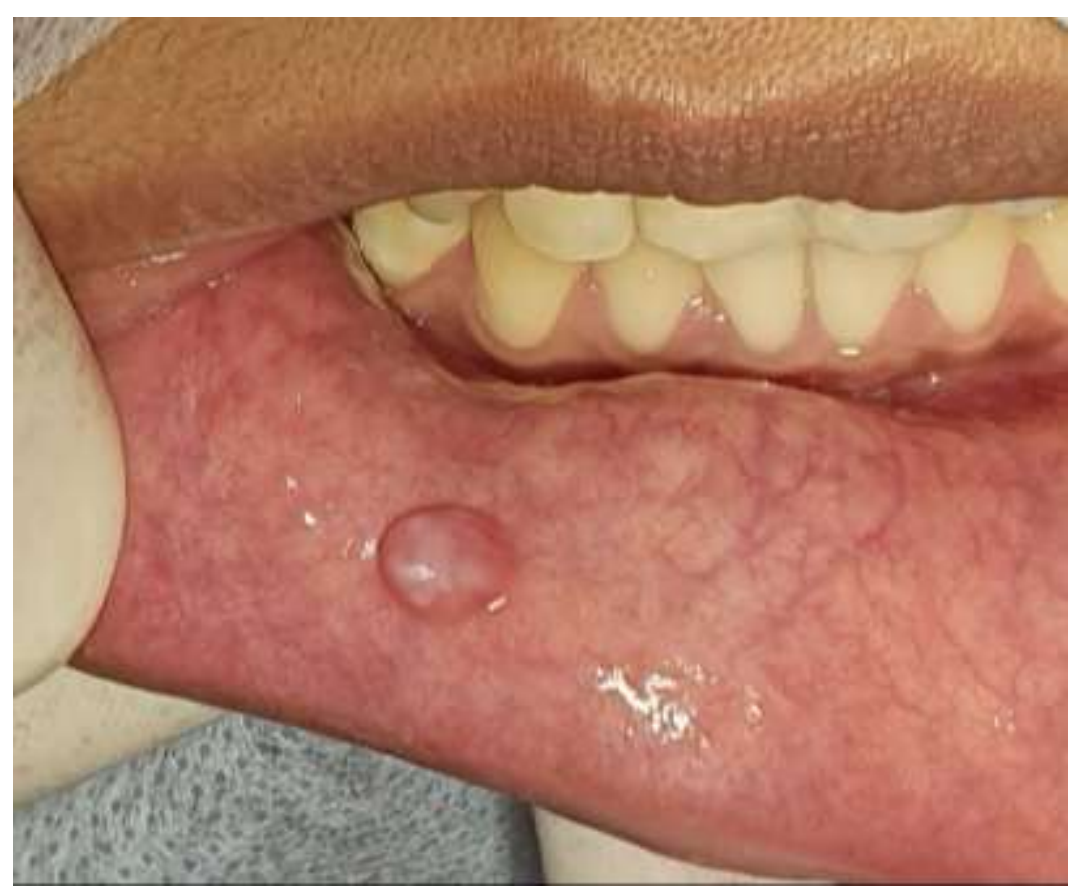

Fonte: Caso clínico dos autores.

Devido ao longo tempo de evolução, a paciente referiu não se recordar de qualquer trauma previamente ao surgimento da lesão. Porém, relatou atrito constante durante a mastigação, gerando desconforto.

Sob diagnóstico clínico de mucocele e com as hipóteses de fibroma e lipoma para o diagnóstico diferencial, o tratamento proposto foi a biópsia excisional, com um prognóstico satisfatório estabelecido para o caso. Após o preparo pré-operatório, realizou-se a anestesia tópica da região com EMLA® por 5 minutos, seguida da anestesia local terminal infiltrativa com mepivacaína 2\% + epinefrina 1:100.000, sob a cautela de evitar o mascaramento da lesão pelo excesso de anestésico local (Figura 2). 
Research, Society and Development, v. 10, n. 11, e331101119465, 2021

(CC BY 4.0) | ISSN 2525-3409 | DOI: http://dx.doi.org/10.33448/rsd-v10i11.19465

Figura 2 - Aplicação de anestesia local terminal infiltrativa submucosa.

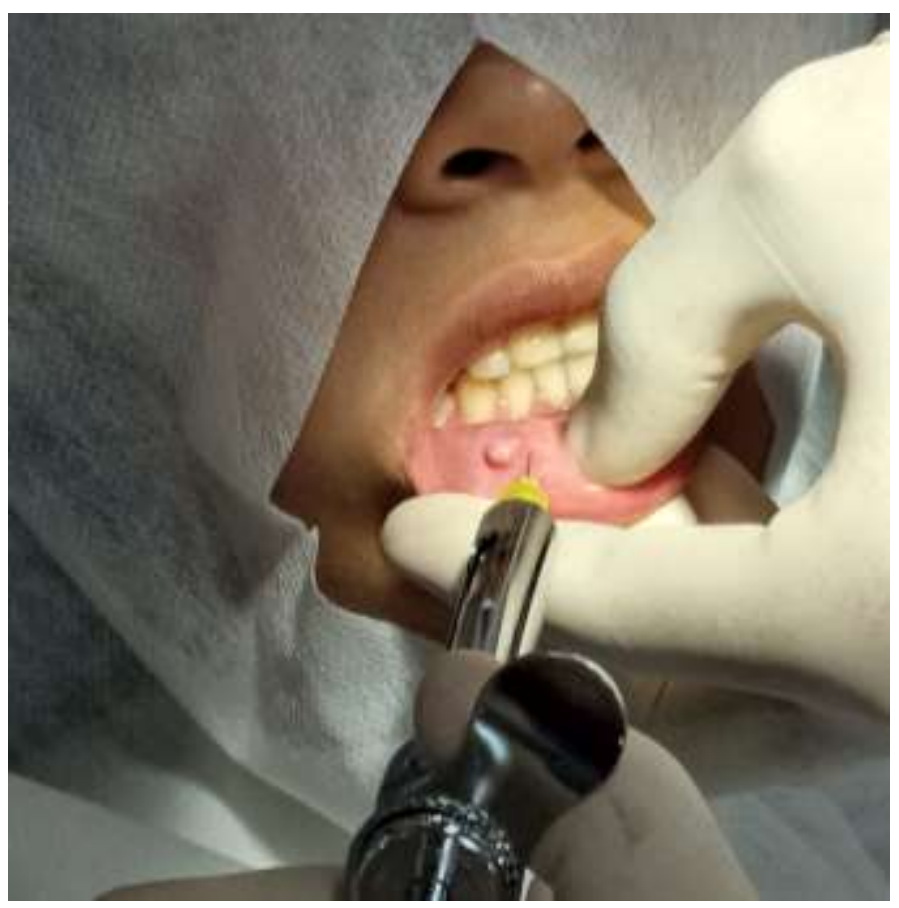

Fonte: Caso clínico dos autores.

A demarcação e incisão mucosa foi realizada com um punch de $6 \mathrm{~mm}$ de diâmetro, que envolveu toda a lesão (Figura 3).

Figura 3 - Posicionamento do punch sobre a lesão para incisão de mucosa e submucosa.

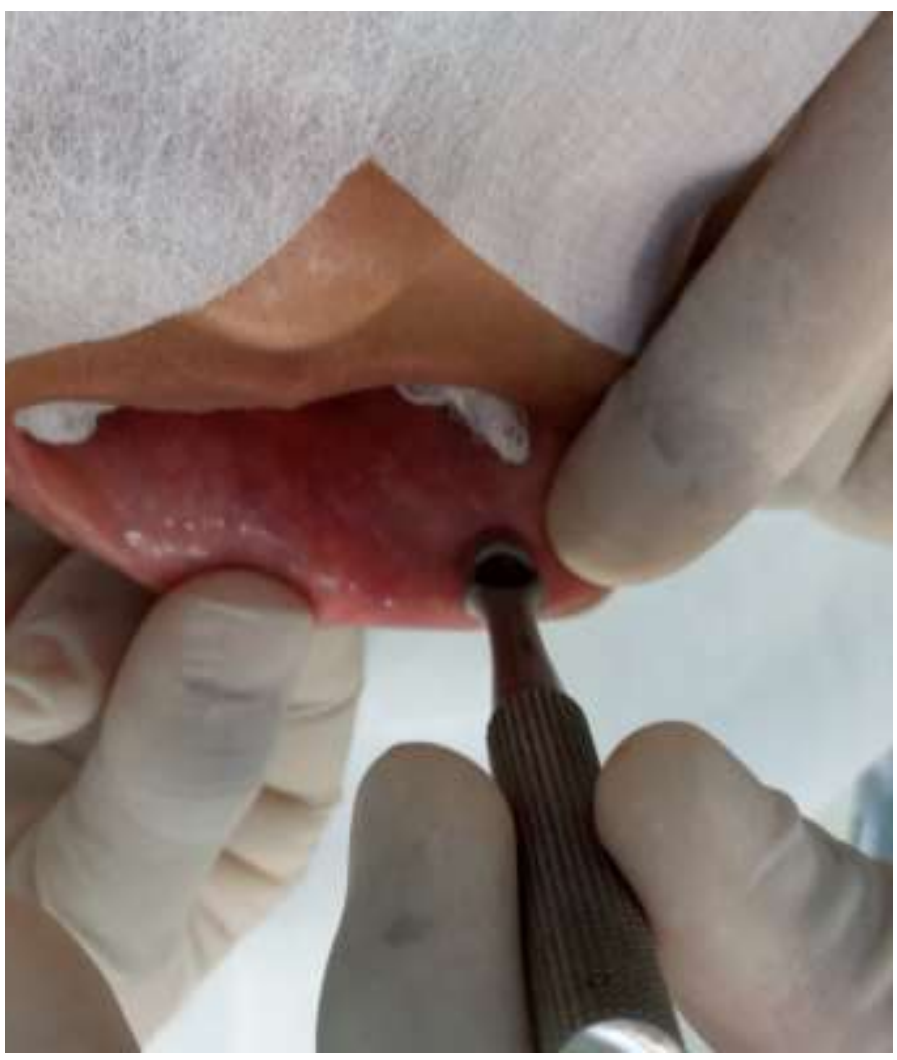

Fonte: Caso clínico dos autores. 
A seguir, realizou-se a divulsão tecidual submucosa com o auxílio de uma tesoura íris para exposição e exérese das glândulas salivares menores associadas para se evitar recidiva (Figura 4).

Figura 4 - Divulsão tecidual com teoura íris.

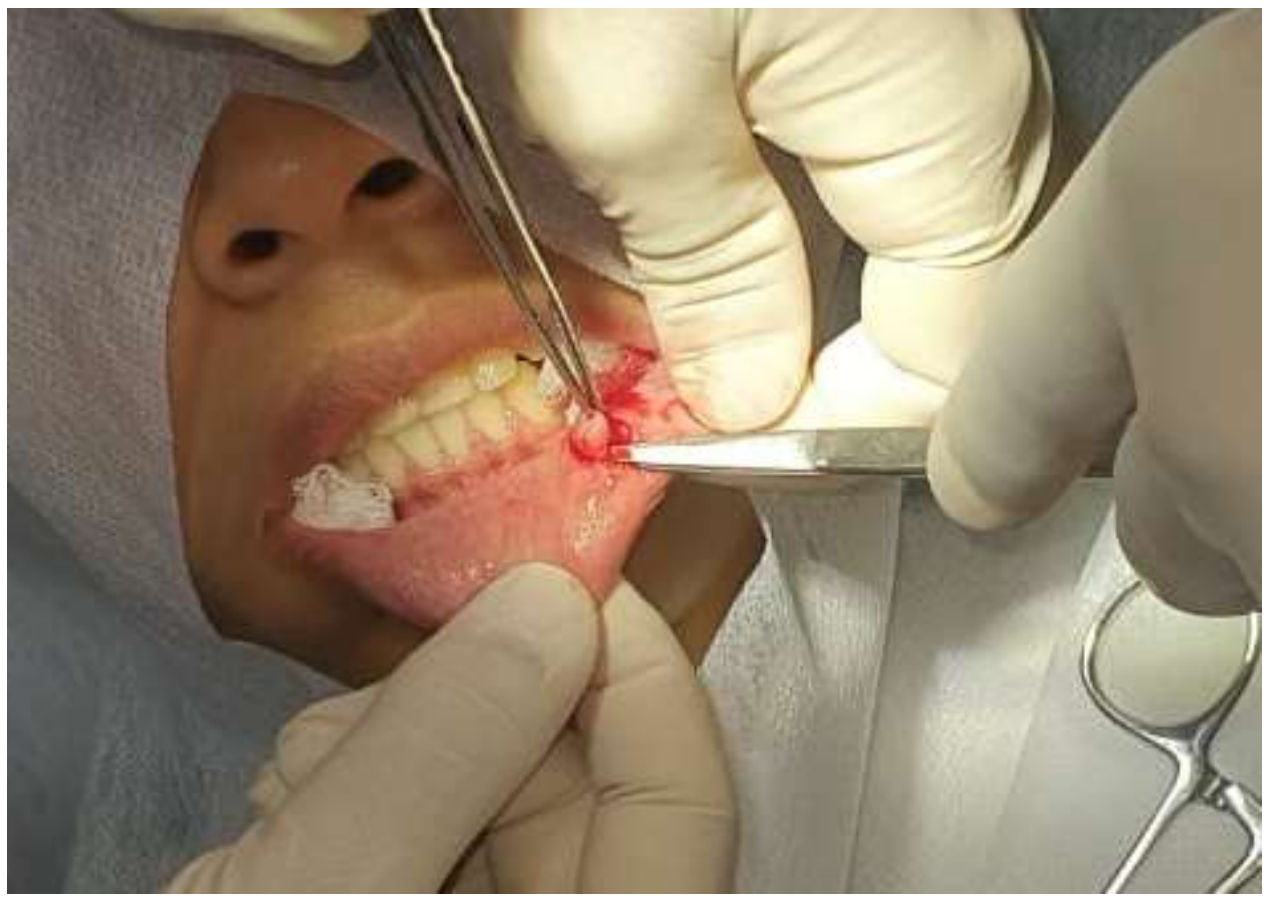

Fonte: Caso clínico dos autores.

Subsequentemente, o procedimento foi finalizado com a revisão da hemostasia e sutura com fio de seda 4.0, utilizando-se de pontos interrompidos (Figura 5).

Figura 5 - Aspecto final da região operada após a sutura, com boa hemostasia.

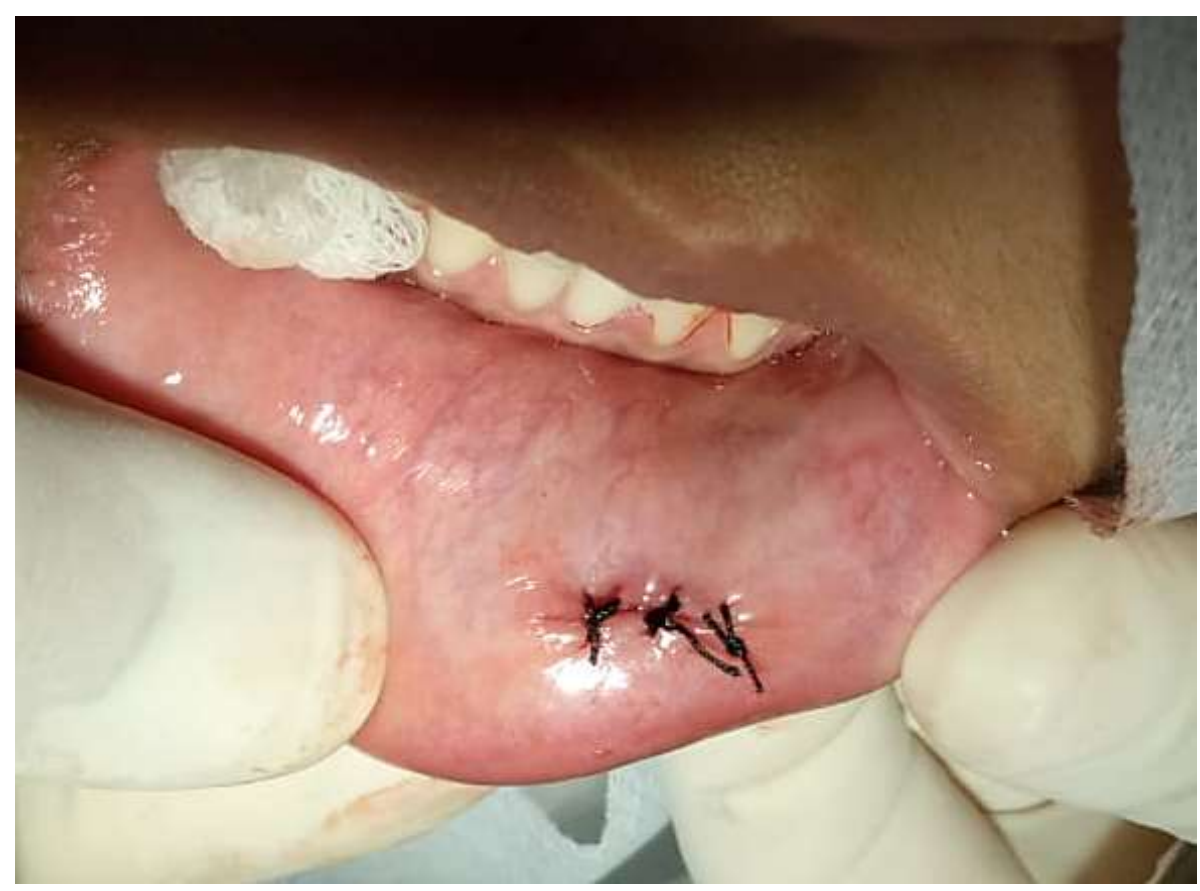

Fonte: Caso clínico dos autores. 
O espécime obtido foi mantido em solução de formol a $10 \%$ e enviado para análise histopatológica. O pós-operatório de 21 dias evidenciou bom aspecto cicatricial, sem qualquer comprometimento local (Figura 6).

Figura 6 - Aspecto pós-operatório aos 21 dias, evidenciando adequado processo cicatricial.

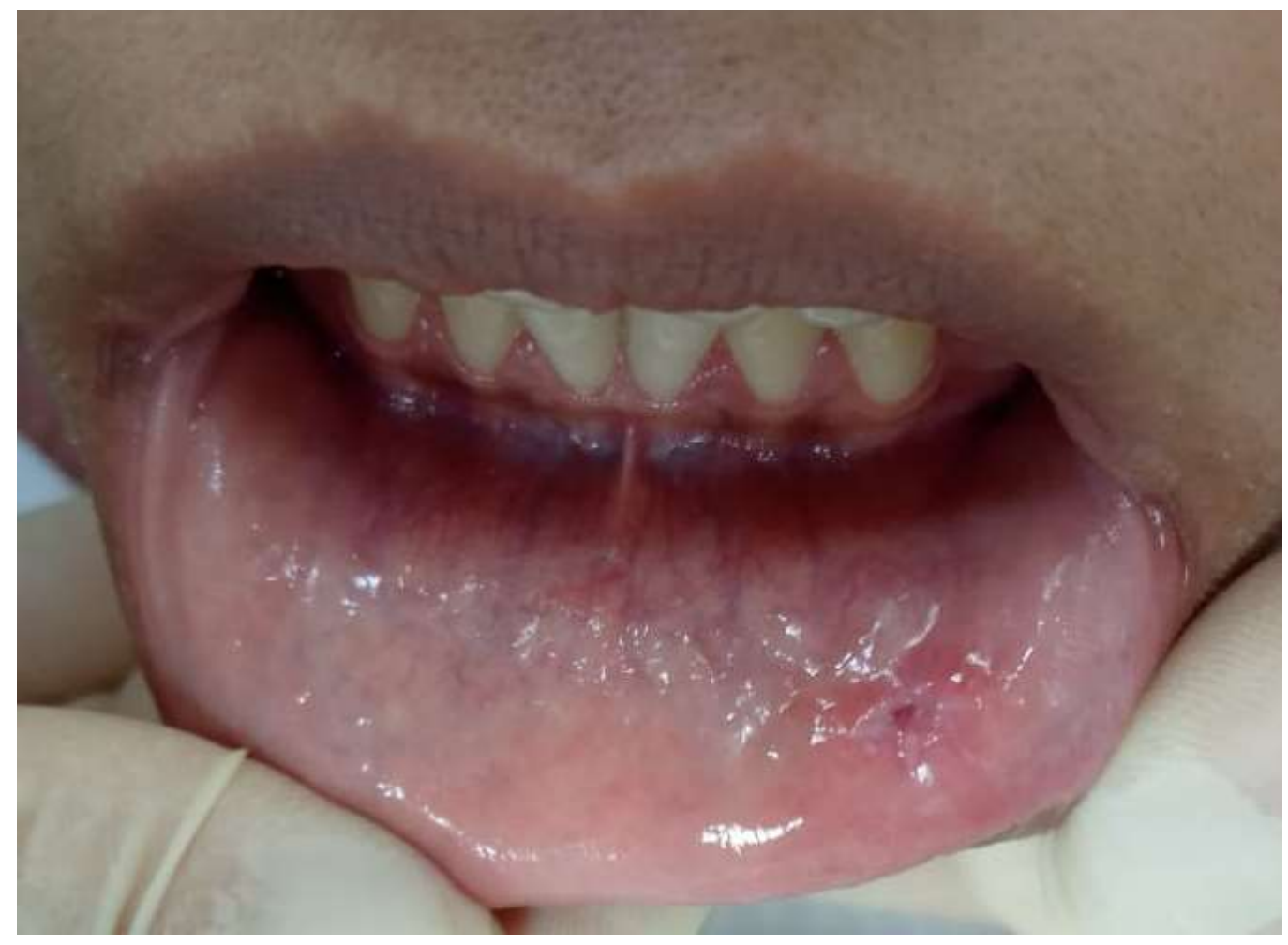

Fonte: Caso clínico dos autores.

O laudo obtido do exame histopatológico, confirmou o diagnóstico de mucocele, onde foi possível observar glândula salivar menor, cavidade com material hialino e infiltrado inflamatório crônico compatível com o diagnóstico de mucocele (Figura 7). 
Figura 7 - Imagem do exame histopatológico, evidenciando glândula salivar menor, cavidade com material hialino e infiltrado inflamatório crônico compatível com o diagnóstico de mucocele.

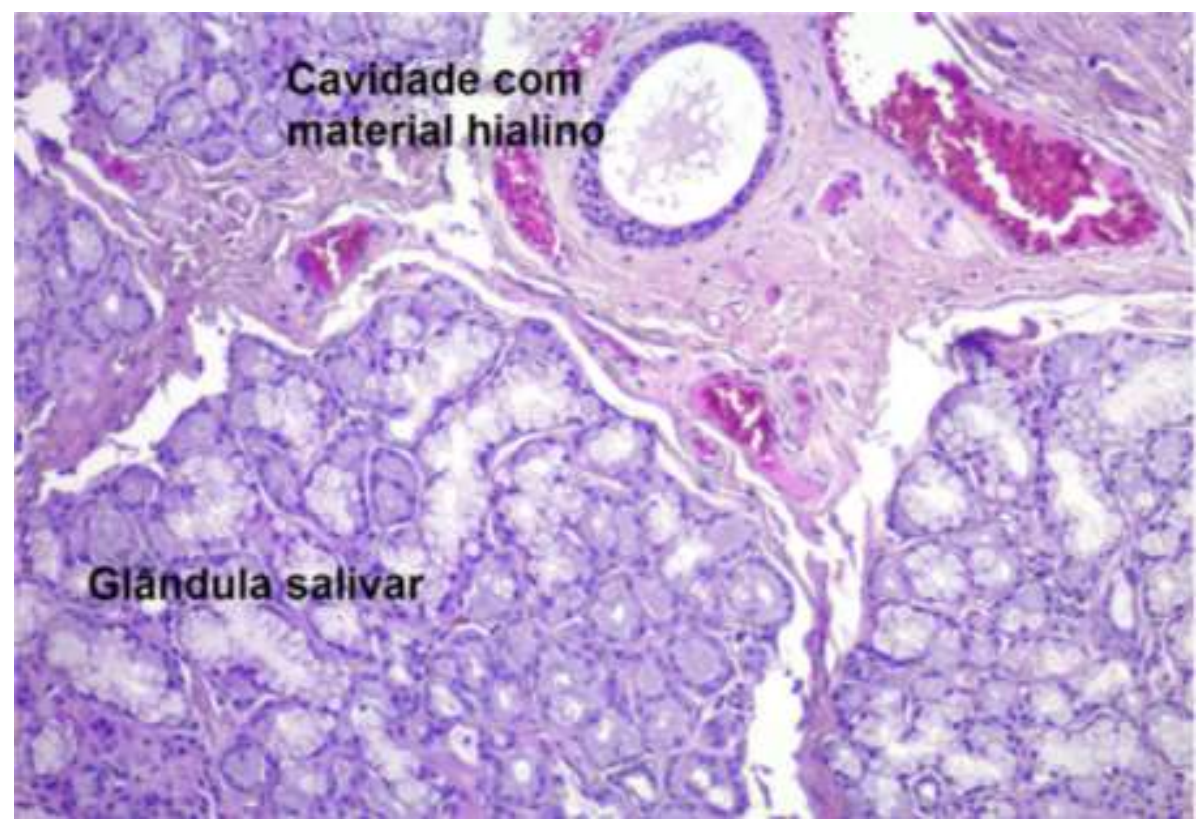

Fonte: Caso clínico dos autores.

\section{Discussão}

As mucoceles, consideradas as lesões benignas mais comuns a se desenvolver na cavidade oral, acometem as glândulas salivares menores. São decorrentes de traumas onde ocorre o rompimento ou obstrução do ducto da glândula salivar menor e posterior derramamento de material mucoso aos tecidos adjacentes. Esta lesão pode se apresentar em vários sítios onde o epitélio apresenta menos queratina. Os locais mais afetados são: mucosa jugal, assoalho bucal (rânula), língua, e na maioria dos casos em lábio inferior, conforme o caso clínico exposto (Santos, Corrêa, Corrêa, 2013; Nascimento et. al, 2014; Giraddi, Saifi, 2016).

Geralmente, a mucocele oral é descoberta pelo cirurgião dentista em exames de rotina ou pelo próprio paciente que percebe um aumento volumétrico ou bolha flutuante e indolor. Sua evolução é lenta e a sua coloração pode variar dependendo da profundidade da lesão. A história clínica do paciente e a aparência da lesão são fundamentais para estabelecer o diagnóstico, mas é possível que sejam confundidas com outros processos patologicos, como o fibroma, e o granuloma piogênico que podem também advir de lesões traumáticas, sendo assim, é imprescindível uma análise histopatológica para confirmar o diagnóstico (Santos, Corrêa, Corrêa 2013; Bezerra et al. 2016; Choi et al. 2019; Neves et al. 2020; Peña et al. 2020; Brooks, Schwartz \& Basile, 2016).

Para a escolha da melhor abordagem terapêutica para o tratamento é necessário levar em consideração a idade do paciente, estado físico ou emocional, e a resistência a procedimentos invasivos. Dentre os tratamentos, a excisão cirúrgica é a principal escolha, pois reduz consideravelmente o risco de recidivas, entretanto, é necessário que haja a dissecção da lesão até a camada muscular e a remoção das glândulas salivares menores associadas circundantes à lesão (Oliveira et al, 2018).

A biópsia com auxílio do punch, como feita no caso relatado, é um procedimento simples, rápido, barato e seguro para o diagnóstico de lesões na mucosa oral com um punch. As vantagens incluem o baixo índice de morbidade pós-cirúrgica, a necessidade de suturas é incomum e a técnica pode ser aplicada a qualquer superfície acessível ao punch. As possíveis desvantagens a serem observadas incluem a dificuldade de se obter tecido adequado e representativo mais profundo do que a lâmina própria superficial; a mucosa se mantém móvel e não pode ser bem suportada em regiões como o palato mole e o 
assoalho de boca, o que poderia impedir a aplicação da técnica; a técnica deve ser utilizada com cautela quando as lesões recobrem significativas estruturas submucosas; o acesso ao região vestibular do rebordo na porção posterior de maxila e no aspecto lingual anterior da mandíbula é dificultoso (Lynch \& Morris, 1990). A análise histopatológica do espécime enviado foi de fundamental importância para a obtenção do diagnóstico definitivo, evidenciando microscopicamente estruturas características de um quadro de mucocele, como a presença de glândula salivar menor, cavidade com conteúdo hialino e infiltrado inflamatório (Nascimento, Azevedo, Barros \& Takahara-Júnior, 2014).

Apesar da facilidade da técnica, que permite a rotação do punch para frente e para trás, entre os dedos, a biópsia em tecido labial, como no caso apresentado, exige o delicado controle de profundidade e o suporte tecidual externo com auxílio dos dedos do cirurgião. Além disso, a liberação da peça biopsiada, deve ser realizada com uma tesoura íris curva e, se tratando de mucocele, a dissecção até a camada muscular e remoção das glândulas salivares menores associadas se procede de modo semelhante, (Lynch \& Morris, 1990; Gill, 1996).

Embora a terapia cirúrgica convencional seja a principal opção terapêutica, pode-se lançar mão de outras abordagens, como a crioterapia, cirurgia a laser, a micromarsupialização, utilização de corticosteroides e escleroterapia (Oliveira et al, 2018). A crioterapia utiliza a aplicação do frio por meio do gás óxido nitroso ou spray de nitrogênio líquido com temperaturas abaixo de $-20^{\circ} \mathrm{C}$ (Farah \& Savage, 2006; Gaddam \& Sharma, 2010). O laser de diodo apresenta benefícios significativos para a exérese de lesões semelhantes, como a boa hemostasia e menor tempo operatório, porém é necessário o investimento material (Ganguly, Mukherjee \& Pal, 2015). A micromarsupialização é considerada uma técnica minimamente invasiva, e a maioria dos casos pode ser realizada sob anestesia tópica isolada, rapidamente, sendo bem tolerado pelo paciente, onde visa drenar a saliva retida na lesão através da colocação de um fio de sutura ao longo do maior diâmetro da lesão estabelecendo uma comunicação entre a cavidade e o meio oral, levando ao desaparecimento da lesão, considerada uma técnica adequada para crianças (Girish, Giraddi, Aamir, Malick \& Saifi, 2017). A aplicação intralesional de corticosteroides e a escleroterapia utilizam agentes como betametasona e o tetradecil sulfato de sódio respectivamente, o que permite a completa resolução da lesão sem a necessidade de cirurgia (Oliveira et al, 2018; Shetty, Rao, \& Pai, 2018).

\section{Conclusão}

A abordagem terapêutica adotada, biópsia com auxílio do punch, apesar de mais invasiva, comparada as demais terapias disponíveis, apresenta-se com excelente custo benefício, permite um rápido tempo cirúrgico, acesso muito bem delimitado e com bordas regulares e além disso, mostrou-se eficaz na resolução do caso relatado, sem complicações transoperatórias, pós-operatórias ou recidivas. Nesse sentido, o estudo apresentado ainda contribui para que futuras comparações sejam realizadas entre a abordagem com bisturi ou punch dentro da excisão cirúrgica convencional.

\section{Referências}

Bansal, S., Verma, D. K., Goyal, S., \& Rai, M. (2017). Comparison of Micromarsupialization and Modified Micromarsupialization for the Management of Mucocoele of Lower Lip: A Prospective Randomized Clinical Trial. Journal of maxillofacial and oral surgery, 16(4), 491-496. https://doi.org/10.1007/s12663-017-1004-0

Bezerra, T. M., Monteiro, B. V., Henriques, Á. C., de Vasconcelos Carvalho, M., Nonaka, C. F., \& da Costa Miguel, M. C. (2016). Epidemiological survey of mucus extravasation phenomenon at an oral pathology referral center during a 43 year period. Brazilian journal of otorhinolaryngology, 82(5), 536-542. https://doi.org/10.1016/j.bjorl.2015.09.013

Brooks, J. K., Schwartz, K. G., \& Basile, J. R. (2016). Superficial Mucocele of the Ventral Tongue: Presentation of a Rare Case and Literature Review. Journal of oral and maxillofacial surgery: official journal of the American Association of Oral and Maxillofacial Surgeons, 74(6), 1175-1179. https://doi.org/10.1016/j.joms.2015.11.025

Choi, Y. J., Byun, J. S., Choi, J. K., \& Jung, J. K. (2019). Identification of predictive variables for the recurrence of oral mucocele. Medicina oral, patologia oral y cirugia bucal, 24(2), e231-e235. https://doi.org/10.4317/medoral.22690 
Gill D. (1996). Two simple treatments for lower lip mucocoeles. The Australasian journal of dermatology, 37(4), 220. https://doi.org/10.1111/j.14400960.1996.tb01061.x

Farah C. S., \& Savage N. W. (2006). Cryotherapy for treatment of oral lesions. Aust Dent J. 51(1):2-5.

Gaddam S., \& Sharma P. (2010). Advances in endoscopic diagnosis and treatment of Barrett's esophagus. J Dig Dis.; 11(6):323-33.

Ganguly R., Mukherjee M., \& Pal T. K. (2015). Laser excision of a mucocele: a case report. J Int Clin Dent Res Organ; 7(2):168-70.

Jinbu, Y., Kusama, M., Itoh, H., Matsumoto, K., Wang, J., \& Noguchi, T. (2003). Mucocele of the glands of Blandin-Nuhn: clinical and histopathologic analysis of 26 cases. Oral surgery, oral medicine, oral pathology, oral radiology, and endodontics, 95(4), 467-470. https://doi.org/10.1067/moe.2003.51

Lynch, D. P., \& Morris, L. F. (1990). The oral mucosal punch biopsy: indications and technique. Journal of the American Dental Association (1939), 121(1), 145-149. https://doi.org/10.14219/jada.archive.1990.0149

Mendes, B. C., Bonardi, J. P., Silva, L. F., Crivelini M. M., \& Bassi, A.P. F. (2020). Granular cell ameloblastoma in maxilla: update of unusual clinical, radiographic and histological appearance. Research, Society, and Development, 9, 01-09. http://dx.doi.org/10.33448/rsd-v9i7.5194

Mínguez-Martinez, I., Bonet-Coloma, C., Ata-Ali-Mahmud, J., Carrillo-García, C., Peñarrocha-Diago, M., \& Peñarrocha-Diago, M. (2010). Clinical characteristics, treatment, and evolution of 89 mucoceles in children. Journal of oral and maxillofacial surgery: official journal of the American Association of Oral and Maxillofacial Surgeons, 68(10), 2468-2471. https://doi.org/10.1016/j.joms.2009.12.038

Nascimento J. S., Azevedo, R. S., Barros, E. M. V. B., \& Takahama-Júnior, A. (2014). Mucoceles da cavidade oral: análise das características histopatológicas de 42 casos. Rev. Odontol. Bras. Central. 23 (66), 162-165.

Nallasivam, K. U., \& Sudha, B. R. (2015). Oral mucocele: Review of literature and a case report. Journal of pharmacy \& bioallied sciences, 7(Suppl 2), S731S733. https://doi.org/10.4103/0975-7406.163516

Nico, M. M., Park, J. H., \& Lourenço, S. V. (2008). Mucocele in pediatric patients: analysis of 36 children. Pediatric dermatology, 25(3), 308-311. https://doi.org/10.1111/j.1525-1470.2008.00672.x

Oliveira, B. F., Henrique, D. B. B., \& Cruz, J. H. A. (2018). Mucocele oral provocada por mordida acidental: relato de caso. Arch Health Invest (2018) 7(11):455-460. http://dx.doi.org/10.21270/archi.v7i11.3128

Rodrigues, M. T. V., Schueng, F. E. A., Mendes, B. C. Vargas Pinto, J. M. Souza, F. G., \& Noia, F. C. (2021). Facial cellulitis caused by Gyant parotid sialolith: a minimally invasive treatment for a rare occurrence. Research, Society and Development, 10, 01-09. http://dx.doi.org/10.33448/rsd-v10i3.13754

Santos T. S., Martins Filho P. R. S., Menezes F. S., Maia M. C., Carvalho R. W. F., Araújo F. A. C. (2008).Tratamento cirúrgico de mucocele utilizando a técnica de Shira: relato de caso. UFES Rev Odontol.; 10(4): 53-8

Shetty, V. M., Rao, R., \& Pai B, S. (2018). Sclerotherapy in Mucocele: A Novel Therapeutic Approach. Journal of cutaneous medicine and surgery, 22(6), 652-653. https://doi.org/10.1177/1203475418775376

Silva, W. P. P, Barbosa S, Mendes, B. C., Pavelski, M. V., Santos, A. M. S., Silva M. C., Santos, J. M. F., Magro-Filho, O., \& Faverani, L. P. (2021). Clinical and systemic management of myiasis in a patient with advanced Alzheimer's, Disease. Research, Society and Development, 10 , 01-07. http://dx.doi.org/10.33448/rsd-v10i3.13145

Khandelwal, S., \& Patil, S. (2012). Oral mucoceles - review of the literature. Minerva stomatologica, 61(3), 91-99.

Valério, R. A., de Queiroz, A. M., Romualdo, P. C., Brentegani, L. G., \& de Paula-Silva, F. W. (2013). Mucocele and fibroma: treatment and clinical features for differential diagnosis. Brazilian dental journal, 24(5), 537-541. https://doi.org/10.1590/0103-6440201301838 